POS $\quad$ PROCEEDINGS

\title{
Proton flux variations in the inner radiation belt in solar cycle 24
}

\author{
S.Yu. Aleksandrin ${ }^{* \dagger}$ \\ National Research Nuclear University MEPhI (Moscow Engineering Physics Institute) \\ E-mail: syaleksandrin@mephi.rui
}

\section{A.M. Galper}

National Research Nuclear University MEPhI (Moscow Engineering Physics Institute)

E-mail: AMGalper@mephi.ru

\section{S.V. Koldashov}

National Research Nuclear University MEPhI (Moscow Engineering Physics Institute)

E-mail: SVKoldashov@mephi.ru

V.V. Malakhov

National Research Nuclear University MEPhI (Moscow Engineering Physics Institute)

E-mail: SVKoldashov@mephi.ru

V.V. Mikhailov

National Research Nuclear University MEPhI (Moscow Engineering Physics Institute)

E-mail: vvmikhajlov@gmail.com

\section{T.R. Zharaspayev}

National Research Nuclear University MEPhI (Moscow Engineering Physics Institute)

E-mail: masscryegmail.com

This report presents the results of analysis of proton flux variations in the inner radiation belt obtained by ARINA spectrometer. The ARINA satellite experiment was carried out on board the Russian low-orbit satellite Resurs-DK1 (altitude 350-600 km, inclination 70 ${ }^{\circ}$ ) from 2006 till 2016. The spectrometer registered high-energy protons (30-100 MeV) with energy resolution of $10 \%$ and angular resolution of $7^{\circ}$. In this work proton flux variations in the inner radiation belt $(\mathrm{L}=1.14, \mathrm{~B}=0.20 \mathrm{G})$ were analyzed with solar index $\mathrm{F} 10.7$ in various phases of the 24th solar cycle. Proton flux anticorrelates with F10.7 index (coefficient correlation -0.95) and its value varies 5-7 times. Time lag between proton flux and F10.7 index was found to be 5-6 months for total period (2006-2016) and it can decrease to 2-3 months in some periods of the 24th solar cycle.

36th International Cosmic Ray Conference -ICRC2019-

July 24th - August 1st, 2019

Madison, WI, U.S.A.

${ }^{*}$ Speaker.

${ }^{\dagger}$ A footnote may follow. 


\section{Introduction}

The inner radiation belt (RB) consists mostly of protons with energies of several tens of $\mathrm{MeV}$. The proton fluxes exceed a thousand times the background and can substantially vary in time. There are various reasons influencing particle fluxes in the RB, as external (changing solar activity) and internal (changing of geomagnetic field).

The study of fluxes has both scientific and practical interest $[1,2,3]$.

\section{Instruments}

The main part of the ARINA spectrometer [4] is a multi-layer scintillation detector. The instrument measures fluxes of electrons with energies of 3-30 MeV and protons with energies of 30-100 MeV. Spectrometer energy resolution for electrons is $15 \%$, and for protons - about $10 \%$. The angular resolution is $\pm 7^{\circ}$.

It is designed as a pyramidal stack of scintillation layers. Each layer is viewed by pair of photomultiplier tubes and amplitude of signal allows to select type of registered particles (the pulse amplitude from relativistic electrons is much less than from nonrelativistic protons). Energy of detecting particle determines by their range.

Spectrometer ARINA was installed on board Resurs-DK1 satellite [5], which was launched in orbit (an inclination of $70^{\circ}$ and $350-600 \mathrm{~km}$ altitude) in June 2006. The satellite's orbit was changed to a circular with altitude about $600 \mathrm{~km}$ in September 2010. The satellite was continuously operated until January 2016 [6].

\section{Fluxes of protons in the South Atlantic Anomaly region}

Proton fluxes in the South Atlantic Anomaly region can have a short-term variations (cause by solar flares, CME, etc.) and long-term changes (for instance solar cycles). In this paper we studied the flux of protons with energies from 30 to $100 \mathrm{MeV}$ during 23rd and 24th solar cycles. The flux vs L-shells in range 1 to 5 were obtained by the ARINA data [7]. Figure 1 shows that the lower boundary of the RB is observed on the L-shells 1.12-1.2. The proton flux sharply increases ( $10^{4}$ times for $\mathrm{L}=1.2$ ). For the next region ( $\mathrm{L}$ from 1.2 to 2.0 , the $\mathrm{RB}$ ) is characterized by a high proton flux. It is weakly dependent on the L-shell. Figure 1 shows that the proton flux strongly decreases with $\mathrm{L}$-shells in the outer boundary of th $\mathrm{RB}(\mathrm{L}=2.0-3.0)$. Then its slow growth is observed, determined by albedo protons.

Figure 1 shows the data for 2009 and 2014, which are periods of minimum and maximum of solar activity, respectively. The relative flux difference $\delta \mathrm{I}$ for these periods was analyzed for various L-shells.

$$
\delta I=\frac{I_{2009}(L)-I_{2014}(L)}{I_{2014}(L)}
$$

where $\mathrm{I}_{2009}$ and $\mathrm{I}_{2014}$ - the corresponding proton fluxes. The results are shown in the Figure 2. It can be seen that in different zones the proton flux varies at different. For L-shells range 1.12-1.2 $\delta \mathrm{I}$ varies the strongest. Proton flux in the $\mathrm{RB}(\mathrm{L}=1.2-2.0)$ is almost steady. $\delta$ I significantly weakly changes in the outer boundary of the RB $(\mathrm{L}=2.0-3.0)$ than in inner zone of the RB. 


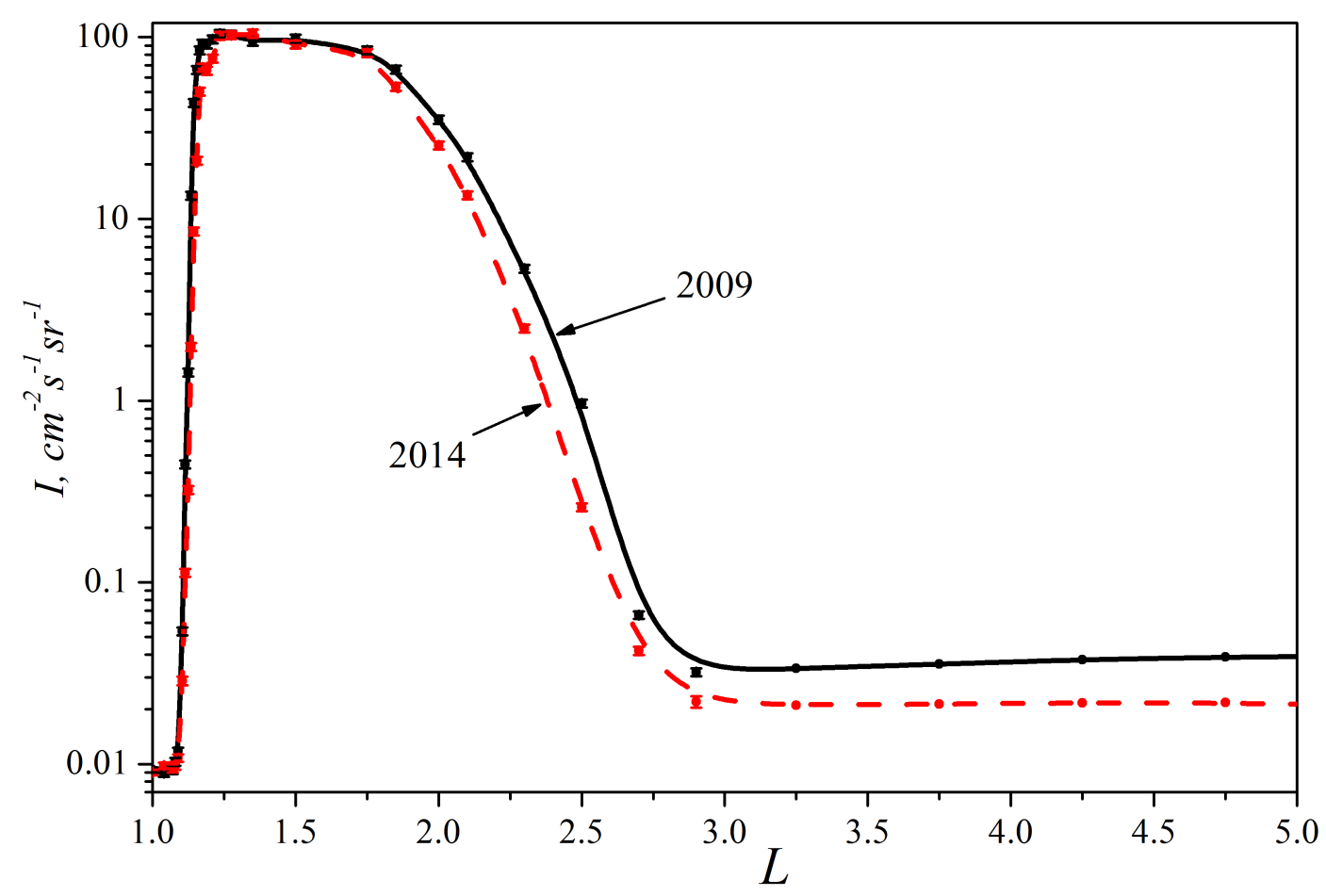

Figure 1: Proton flux as a function of L-shell for minimum (2009) and maximum (2014) of solar activity.

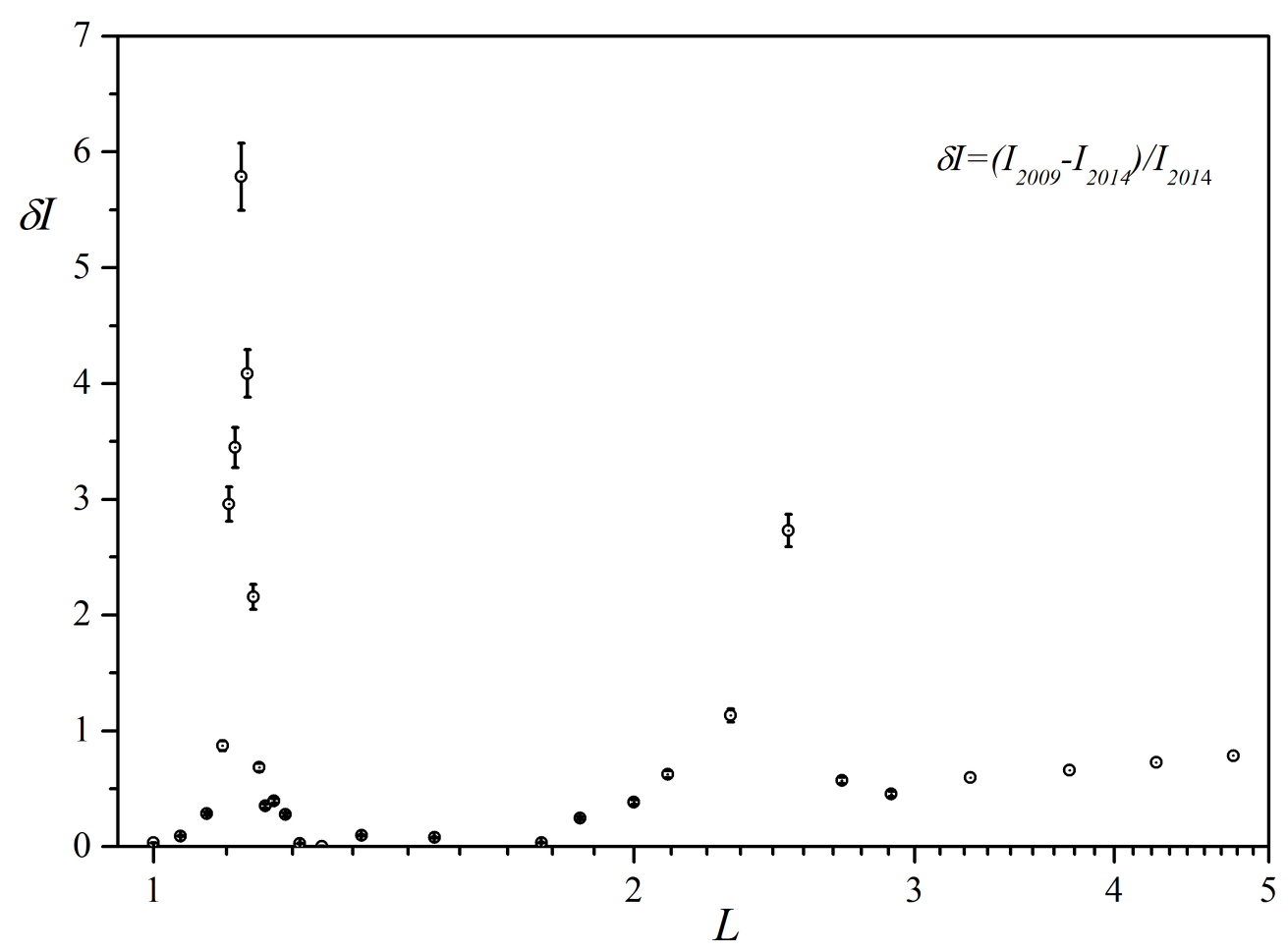

Figure 2: The relative flux difference $\delta \mathrm{I}$ as function of L-shell. 
The Figure 3 shows the proton fluxes as a function of B (the magnitude of the Earth's magnetic field in proton mirror points) for the proton energy $30-100 \mathrm{MeV}$ on the L-shell 1.5. The figure presents the data for 2009 (minimum of solar activity) and 2014 (maximum of solar activity). The flux of minimum is higher than in the period of maximum of solar activity.

And it is shown that the proton flux significantly higher in minimum of solar activity. Life time of protons in inner RB depends on altitude of mirror points of particles. And for low altitude or high value $\mathrm{B}_{m}$ residual density of Earth's atmosphere changes during solar activity cycle. So proton flux depending on atmosphere density changes during solar cycle (Figure 3).

$\mathrm{L}=1.5$

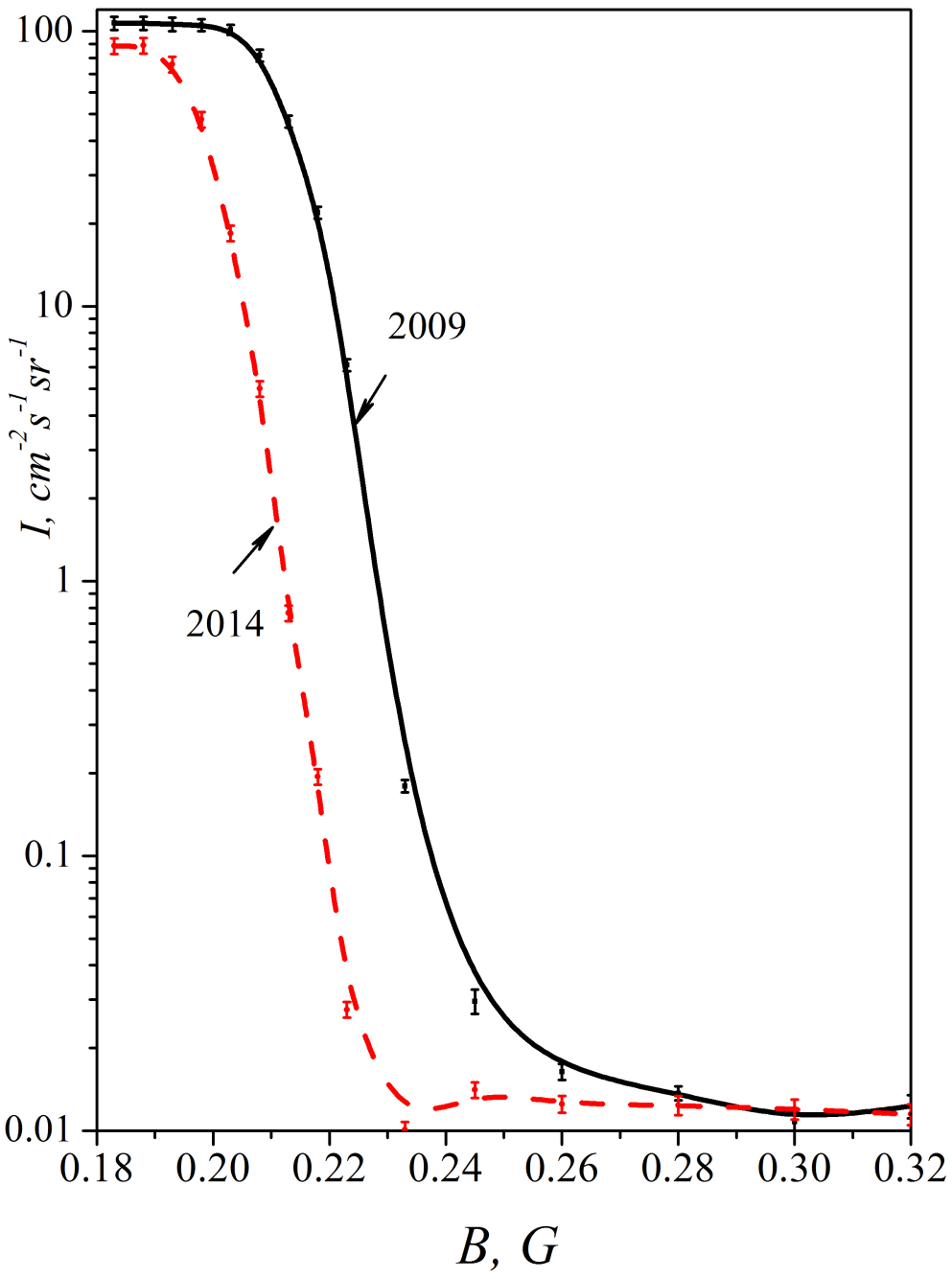

Figure 3: Dependence of proton flux on magnitude of magnetic field ( $\mathrm{L}=1.5)$.

Altitude mirror points for charged particles decreases to the minimum values in the region of the SAA $(300 \mathrm{~km})$ during their drift around Earth.. Atmosphere density correlates with solar activity on this altitude $\left(300^{\circ} \mathrm{E}, 300 \mathrm{~km}\right)$ according to MSISE-90 the Earth's atmosphere density model (Fig. 4) [8]. When drifting particles descent lower in the region of the SAA, they inter- 
act with residual atmosphere lost their energy by ionizing and scatter by it. This defines proton flux decrease on lower border of radiation belt during period of maximum solar activity. Earth atmosphere heats up and expand, its density increases in regions where trapped charged particles drifting around Earth descent the most strong. Particles fluxes drop during solar maximum period respectively.

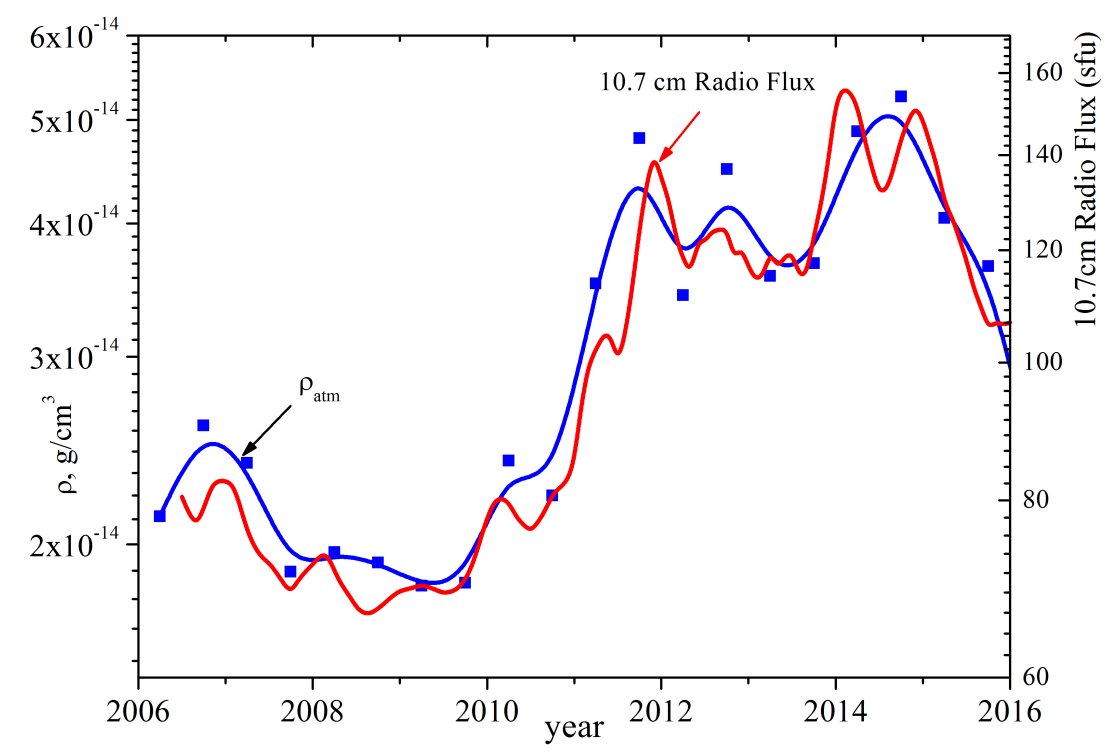

Figure 4: Atmospheric density (MSIS-E90) and solar activity index $10.7 \mathrm{~cm}$ during the observation period.

Figure 5 shows a comparison of the proton flux for selected L-shell, B and solar activity index $10.7 \mathrm{~cm}$ during the solar cycle phases. For $\mathrm{L}=1.13-1.14$ and $\mathrm{B}=0.202-0.207 \mathrm{G}$ proton fluxes in the solar maximum is several times less than in the minimum of solar activity.

It is evident that during the phase of solar maximum, characterized by the maximum of F10.7 index, trapped proton flux decreased in 5-7 times in comparison with the value of the quiet Sun period in the phase of solar minimum. The correlation between the F10.7 index and the proton flux was analyzed. The correlation coefficient $(\mathrm{R})$ was determined by the following formula:

$$
R=\frac{\sum(F 107-\overline{F 107}) \cdot\left(I_{p}-\overline{I_{p}}\right)}{\sum(F 107-\overline{F 107})^{2} \cdot\left(I_{p}-\overline{I_{p}}\right)^{2}}
$$

where $\mathrm{F} 107$ - solar activity index $10.7 \mathrm{~cm}, \mathrm{I}_{p}$ - proton flux for $\mathrm{L}=1.13-1.14$ and $\mathrm{B}=0.202$ $0.207 \mathrm{G}$.

Time lag (Fig.6) between proton flux and F10.7 index was found to be 5-6 months for total period (2006-2016). This delay is less than in the following work [2] but almost coincides with the value in the work [3].

\section{Conclusions}

The results of analyzing data from satellite experiment ARINA are presented. There are several regions of proton flux for inner radiation belt (inner, central and outer zones) where proton 


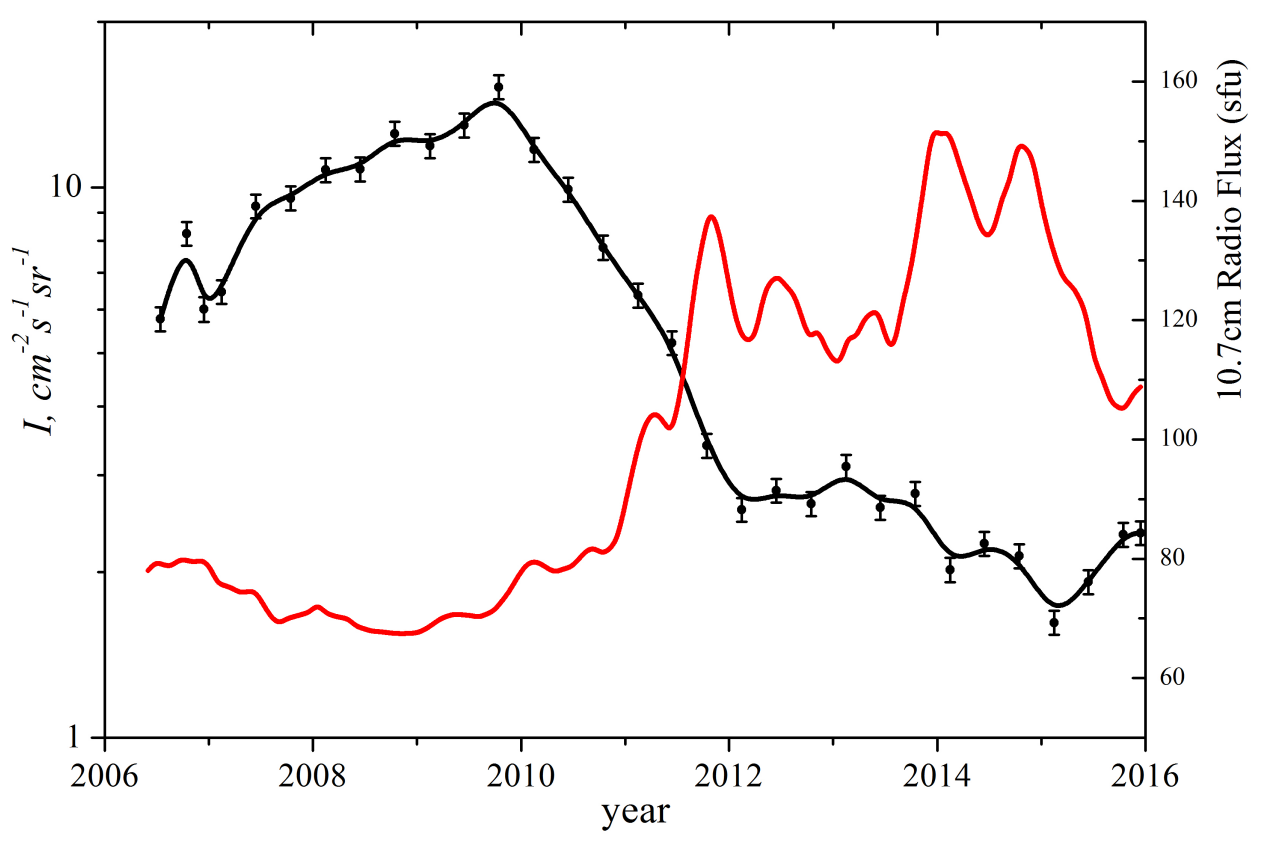

Figure 5: The proton flux low boundary of RB and solar activity index 10.7 during solar cycle 23 and 24 .

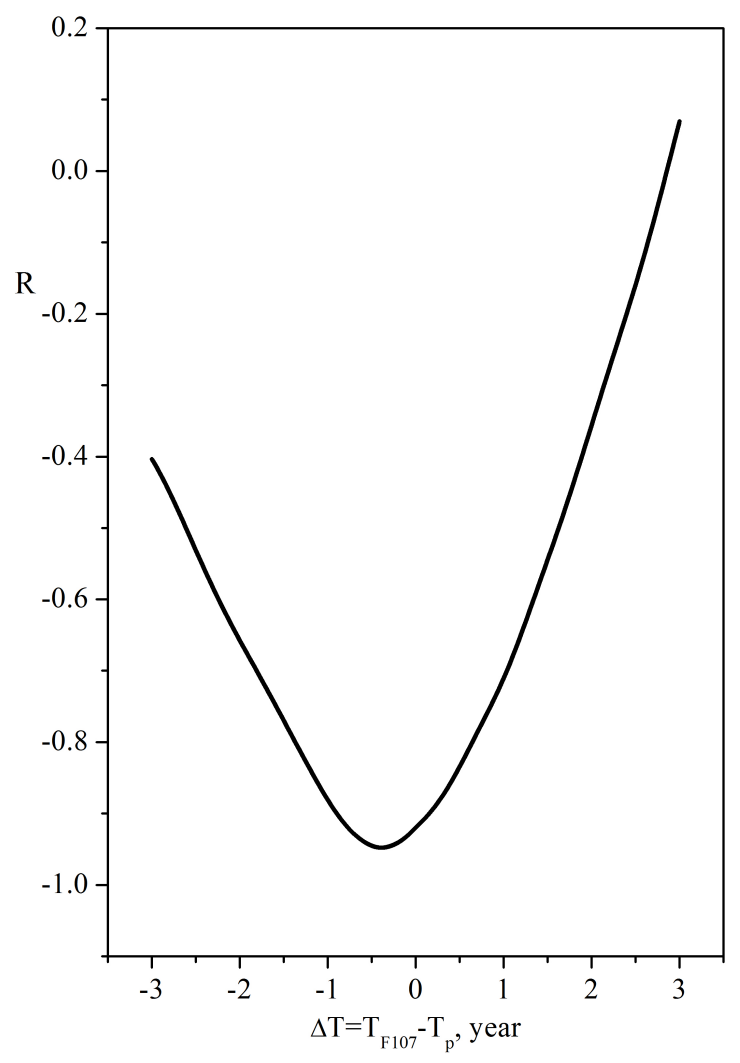

Figure 6: Correlation coefficient between $\mathrm{F} 10.7$ index and proton flux as a function of the lag $\triangle \mathrm{T}=\mathrm{T}_{F 107}-\mathrm{T}_{p}$. 
fluxes vary differently depending on the phase of the solar cycle. The proton flux can increase in dependence on $\mathrm{L}$-shell up to 5-7 times with time lag 5-6 months $(\mathrm{L}=1.14)$ during the Solar minimum.

\section{Acknowledge}

This work was supported by the Russian Ministry of Education and Science, project No 3.2131.2017.

\section{References}

[1] T.P. Dachev, B.T. Tomov, Yu.N. Matviichuk, Pl.G. Dimitrov, N.G. Bankov High dose rates obtained outside ISS in June 2015 during SEP event. Life Sciences in Space Research Vol. 9 (2016) 84-92

[2] Tsvetan P. Dachev South-Atlantic Anomaly magnetic storms effects as observed outside the International Space Station in 2008âĂŞ2016, Journal of Atmospheric and Solar-Terrestrial Physics 179 (2018) 251-260

[3] N. V. Kuznetsov, N. I. Nikolaeva, and M. I. Panasyuk Variation of the trapped proton flux in the inner radiation belt of the earth as a function of solar activity. Cosmic Research, 2010, Vol. 48, No. 1, pp. 80-85., 2010

[4] A.V. Bakaldin, A.G. Batischev, A.M. Galper, L.A. Grishantseva, S. V. Koldashov, P. Yu. Naumov, V. Yu. Chesnokov, and V. A. Shilov. Satellite experiment ARINA for studying seismic effects in the high energy particle fluxes in the Earth's magnetosphere. Cosmic Research. Vol 45. No. 5. p. 471, 2007

[5] V. Bakaldin, A. G. Batischev, M. A. Bjeumikhova, et. al. Instrumentation for experimental recording of bursts of high-energy charged particles in nearEarth space, Bulletin of the Russian Academy of Sciences: Physics 2005, vol. 69, no. 6, p. 918.

[6] http://eng.ntsomz.ru/about/deyatelnost ${ }_{c}$ entra

[7] S. Yu. Aleksandrin, A. M. Galper Spatial and temporal variations of proton fluxes in the Earth's inner radiation belt during a solar cycle. Bulletin of the Russian Academy of Sciences: Physics May 2019, Volume 83, Issue 5, pp 582-583

[8] https://ccmc.gsfc.nasa.gov/modelweb/models/msis itmo.php 